\title{
Regularities of Nanofocusing of the Electromagnetic Field of a Fractal Rough Surface
}

\author{
Nikolay N. Grinchik¹, Galina M. Zayats², Olga V. Boiprav³, Kirill V. Dobrego ${ }^{4}$, Volha A. Prykhodzka \\ ${ }^{1}$ A.V. Luikov Heat and Mass Transfer Institute of the National Academy of Sciences of Belarus, Minsk, Belarus \\ ${ }^{2}$ Institute of Mathematics of the National Academy of Sciences of Belarus, Minsk, Belarus \\ ${ }^{3}$ Belarusian State University of Informatics and Radioelectronics, Minsk, Belarus \\ ${ }^{4}$ Belarusian National Technical University, Minsk, Belarus \\ ${ }^{5}$ Belarusian State University, Minsk, Belarus \\ Email:nngrin@yandex.ru, zayats@im.bas-net.by, boiprav@tut.by, dobrego@bntu.by, olgaprihodko167@gmail.com
}

How to cite this paper: Grinchik, N.N., Zayats, G.M., Boiprav, O.V., Dobrego, K.V. and Prykhodzka, V.A. (2019) Regularities of Nanofocusing of the Electromagnetic Field of a Fractal Rough Surface. Journal of Electromagnetic Analysis and Applications, 11, 117-133.

https://doi.org/10.4236/jemaa.2019.118008

Received: June 9, 2019

Accepted: August 26, 2019

Published: August 29, 2019

Copyright (C 2019 by author(s) and Scientific Research Publishing Inc. This work is licensed under the Creative Commons Attribution International License (CC BY 4.0).

http://creativecommons.org/licenses/by/4.0/

\begin{abstract}
A consistent physical and mathematical model of the propagation of electromagnetic waves in an inhomogeneous medium with strong discontinuities of the electromagnetic field at the interface of two media, which is a rough surface, was developed. Mathematical modeling of rough surfaces and their profiles was carried out using fractal geometry, which allows us to display the topology of the object as close as possible to reality. For real heterogeneous rough structures, we have developed a through-counting method that takes into account the continuity of the total current at the interfaces of adjacent media, the effect of induced surface charge and surface current. This approach lets one avoid the necessity to set surface impedances depending on the structure of the field being determined and on the material properties.
\end{abstract}

\section{Keywords}

Nonstationary Broadening of the Spectral Line, Fractal Roughness, Surface Charge, Surface Current, Electromagnetic Wave, Soliton-Like Electromagnetic Pulse

\section{Introduction}

Imperfectness of the shielding surface is the main factor determining the loss of the guide electrodynamic structure. It is manifested in the extremity of the specific conductivity of the material and the roughness of the shielding surface, determined by the quality of its processing. The problems of the interaction of the 
electromagnetic field with the interface were considered in a number of works [1]-[34]. A better model for rough surface geometry is fractal geometry. From general physical considerations, it follows that the surface roughness can be neglected if the size of irregularities is much smaller than the depth of the electromagnetic field penetration into the walls of the screen. This condition is usually satisfied at frequencies below hundreds of megahertz. However, if the frequency increases, in the microwave and optic wavebands, the roughness of shielding surfaces becomes the main factor determining the loss of the guide structure, for example, antenna devices [20] [29]. In paper [5], the problem of modeling of the slope of a random surface profile, presented as a sum of a finite number of sinusoids with random phases, is considered. The problem of fluctuations of the surface illumination using the proposed method of modeling slopes is investigated, and as a result, the effectiveness of its application is proved. However, for surfaces with a fractal structure, the method proposed in [5] is not applicable. The problems of accounting for fractal geometry arise during laser microstructuring and polishing of surfaces [15] in technological processes. Methods of laser microstructuring of surfaces are widespread in our time. These include the method of laser smoothing consisting in fast high-temperature exposure and cooling; laser microstructuring of the surface, based on local evaporation of the substance; laser roughness control by focusing laser radiation on the treated surface; laser surface microstructuring using surface electromagnetic waves. The latter method realized in several stages: a surface electromagnetic wave is excited at the interface between two media; after the interference of this wave with the incident wave on the surface, a new intensity distribution of light is formed; then spatial non-uniform surface heating takes place, as a result of which spatial periodic structures arise. Experiments show that this method is one of the most promising.

In our work, an attempt to investigate the interaction of an electromagnetic wave with a real surface having a fractal geometry has been made. The existing classical approaches including the introduction of surface charge and surface current do not allow to investigate the interaction of an electromagnetic wave with a rough fractal surface. Mathematical modeling of fractal rough surfaces using Weierstrass-Mandelbrot formulas, which are continuous but not differentiable, is carried out in this paper. A consistent physico-mathematical model of the propagation of electromagnetic waves in a heterogeneous inhomogeneous system with strong discontinuities of the electromagnetic field at the interface of two media, which is a rough surface, has been developed.

During the numerical simulation of the interaction of an electromagnetic wave with a rough fractal surface, the continuity conditions of the total current and the Dirichlet average theorem in the presence of a strong electromagnetic field discontinuity at the interfaces of inhomogeneous, angular structures are used for the first time. From our point of view, the concept of "surface charge" is an idealization. Since in this case, the electric field strength is infinite at the interfaces. In fact, the electric charge is volumetric, has a finite size, occurs when 
there is a normal component of the electric current and can be summed up with an electrostatic charge. Note that in the presence of angular structures it is fundamentally difficult to correctly formulate the boundary conditions on the tip, where the normal vector has no definite orientation. As is known, the issues of nanofocusing of light on the tip are the central problem of modern near-field optical microscopy. The solution to this problem allows us to achieve a resolution above the Rayleigh diffraction limit of conventional optical instruments. Nano-focusing of light makes it possible to effectively control the flow of information in nano-optics devices [29].

The results of the work can be used to study the influence of the roughness of the shielding surfaces of waveguides for antenna devices and in laser polishing of surfaces.

\section{Fractal Approach to Rough Surfaces}

As previously noted, the surface structure of the waveguide at the micro and nano level plays an important role in determining the properties of the device. Solution of various practical problems related to hydrodynamics, electrodynamics, gas dynamics, etc. requires a very accurate and, most importantly, a realistic description of the structure of interface surfaces. Boundary conditions formulation becomes difficult otherwise.

Simplified descriptions of the surfaces of bodies and of the boundary conditions that form a mathematical model greatly simplify the problem being solved, but, on the other hand, it strongly distorts the reality and in many cases is unfairly approximate.

Excluding the roughness of the surface of a device and taking an object as absolutely smooth leads to a huge number of results and conclusions that are groundless and, generally speaking, invented. It was experimentally shown that the microstructure and nanostructure of the surface greatly affect the results of numerical experiments.

Discrete roughness models [22] [31] were used previously, the parameters of which were chosen experimentally as a result of profile processing, based on microgeometry.

Further, as already mentioned, for modeling rough surfaces, fractal geometry has been used. Its specific feature consists in the constancy of fractal parameters on all scales, their independence of the sample length. The structure of the natural surface is equally fractal at all levels.

We present several formulas by which natural surfaces are modeled.

Weierstrass-Mandelbrot formulas are used for modeling the profile of rough surfaces using the fractal approach.

$$
z(x)=G^{D_{f}-1} \sum_{n=1}^{\infty} \frac{\cos 2 \pi \gamma^{n} x}{\gamma^{\left(2-D_{f}\right)^{n}}}, 1<D_{f}<2, \gamma>1,
$$

where $G$ is the length scale, $D_{f}$ is the fractal dimension, $\gamma$ is the frequency and $\gamma^{n}$ is the frequency spectrum [27]. 
There is another formula for describing the surface profile

$$
z(x)=\sum_{n=-\infty}^{\infty} \gamma^{\left(2-D_{f}\right) n}\left(\cos \varphi_{n}-\cos \left(2 \pi \gamma^{n} x+\varphi_{n}\right)\right)
$$

where $\varphi_{n}$ is the random phase.

The Weierstrass-Mandelbrot formulas are characterized by continuity, autoaffinity, and nondifferentiability.

To describe the roughest surface, we use the function:

$$
\begin{aligned}
z(x, y)= & L\left(\frac{G}{L}\right)^{D_{s}-2}\left(\frac{\ln \gamma}{M}\right)^{\frac{1}{2}} \sum_{m=1}^{M} \sum_{n=0}^{n_{\max }} \gamma^{\left(D_{s}-3\right) n} \\
& \times\left[\cos \varphi_{m, n}-\cos \left(\frac{2 \pi \gamma^{n}\left(x^{2}+y^{2}\right)^{\frac{1}{2}}}{L} \cos \left(\arctan \left(\frac{y}{x}\right)-\frac{\pi m}{M}\right)+\varphi_{m, n}\right)\right], \\
n_{\max }= & \operatorname{int}\left[\ln \left(L / L_{m}\right) / \ln \gamma\right],
\end{aligned}
$$

where $L$ is the sample length, $L_{m}$ is the limiting length, $D_{s}=D_{f}+1$, and $M$ is the number of imposed waves during simulation.

Dealing with rough surfaces that will subsequently represent the interface between two heterogeneous media, we will model their profiles by using Formulas (1) and (2), since the problem will be solved in 2D formulation (see Figures 1-3).

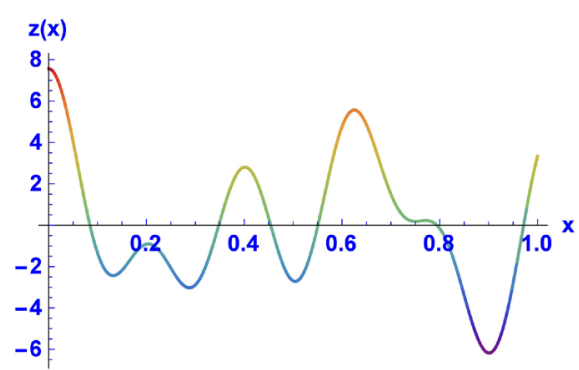

(a)

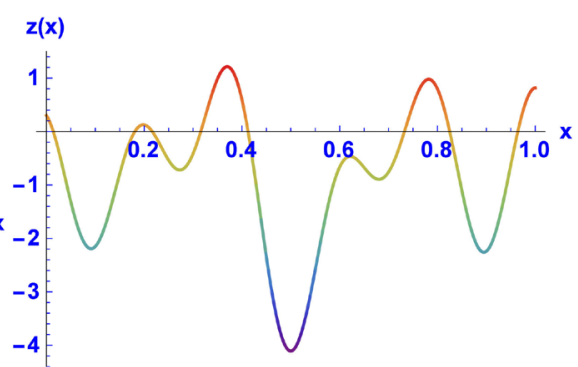

(b)

Figure 1. Profiles of fractal rough surfaces according to: (a) Formula (1); (b) Formula (2) at $M=3, D_{f}=1.9, \gamma=1.7, L=3$ and $G=3$. The summation was made within $[1 ; M]$.

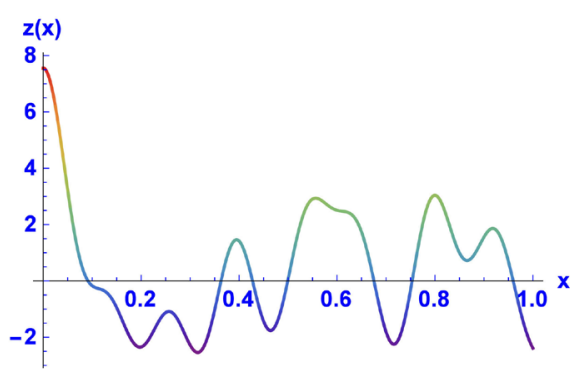

(a)

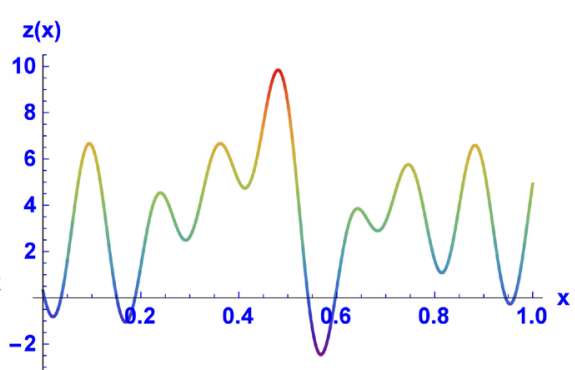

Figure 2. Profiles of fractal rough surfaces according to: (a) Formula (1); (b) Formula (2) at $M=5, D_{f}=1.6, \gamma=1.5, L=4$ and $G=4$. The summation was made within $[1 ; M]$. 
We also model the rough surfaces themselves in $3 \mathrm{D}$ using, for clarity, the fractal approach. We use Formula (3). Quite realistic surfaces are simulated at different parameters. Each surface is provided with a density graph (see Figures 4-6).

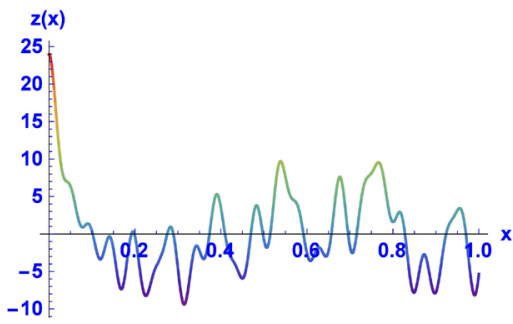

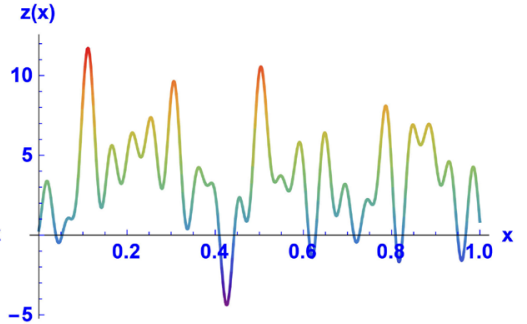

Figure 3. Profiles of fractal rough surfaces according to (a) Formula (1); (b) Formula (2) at $M=9, D_{f}=1.8, \gamma=1.4, L=4$ and $G=5$. The summation was made within $[1 ; M]$.
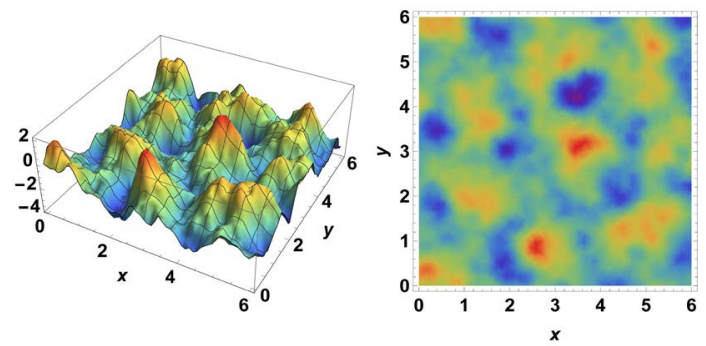

Figure 4. Fractal rough surface and its density graph at $M=7, n_{\max }=6, D_{f}=1.7, \gamma=1.5, L=3$ and $G=3$.
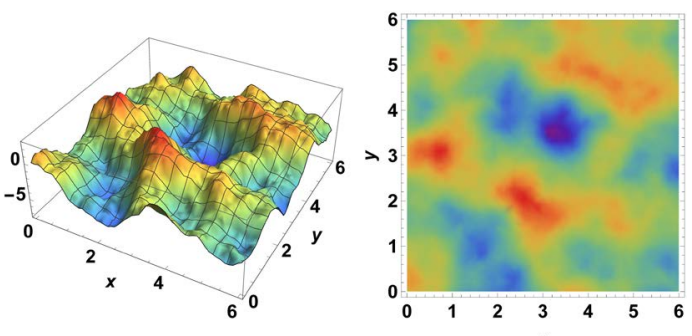

Figure 5. Fractal rough surface and its density graph at $M=12, n_{\max }=12, D_{f}=1.7, \gamma=1.5, L=5$ and $G=6$.
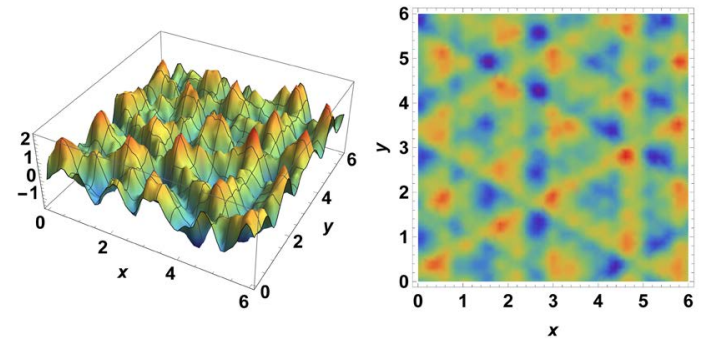

Figure 6. Fractal rough surface and its density graph at $M=3, n_{\max }=3, D_{f}=1.7, \gamma=1.5, L=2$ and $G=2$. 


\section{Development of the Mathematical Model of Electromagnetic Waves Nanofocusing}

Further, we develop a system of differential equations representing a model of the propagation of electromagnetic signals in layered media.

Earlier [7], we developed a consistent physicomathematical model of the propagation of a soliton-like electromagnetic pulse in a heterogeneous inhomogeneous medium with strong discontinuities of the electromagnetic field. The model is based on the reduction of Maxwell's equations to a well-studied wave equation. When specifying an electromagnetic pulse, its amplitude modulation and nonstationary broadening of the spectral line were taken into account. The conditions for pulse matching for the first initial-boundary value problem are obtained. The temporal dispersion of electrical induction is allowed by the function of establishing a signal, taking into account the broadening of its spectral line and integration over the continuous spectrum. With this approach, there is no need to neglect spatial derivatives, as well as to use spatial nonlocal relations to take into account the influence of surface charge, surface current, and spatial dispersion of electrical induction at the interfaces of adjacent media [6] [7] [18] [19].

Electromagnetic radiation propagating in space can encounter various obstacles in its path. Interracting with these obstacles, there is a strong discontinuity of the electromagnetic field at the interfaces, which occurs due to the difference in dielectric constants of the media. On both sides of this surface, the magnetic and electric field strength vectors are continuous and finite [19].

The Maxwell equations, written below in differential form, describe the interaction of a field with a material medium, but cannot be called a complete system, because they do not reflect media properties in the presence of strong electromagnetic field discontinuities at the interface between adjacent media [6] [7] [19].

$$
\begin{gathered}
\boldsymbol{j}_{\mathrm{tot}}=\nabla \times \boldsymbol{H}, \nabla \boldsymbol{D}=\rho, \\
-\frac{\partial \boldsymbol{B}}{\partial t}=\nabla \times \boldsymbol{E}, \nabla \boldsymbol{B}=0 .
\end{gathered}
$$

where $\boldsymbol{j}_{\text {tot }}=\lambda \boldsymbol{E}+\frac{\partial \boldsymbol{D}}{\partial t}, \boldsymbol{B}=\mu \mu_{0} \boldsymbol{H}$ and $\boldsymbol{D}=\varepsilon \varepsilon_{0} \boldsymbol{E}$.

The boundary conditions at the interfaces of two different media in a heterogeneous system are

$$
\begin{gathered}
D_{n_{1}}-D_{n_{2}}=\sigma, \\
E_{\tau_{1}}-E_{\tau_{2}}=0, \\
B_{n_{1}}-B_{n_{2}}=0, \\
\boldsymbol{H}_{\tau_{1}}-\boldsymbol{H}_{\tau_{2}}=\left[\boldsymbol{i}_{\tau} \boldsymbol{n}\right] .
\end{gathered}
$$

where indexes $n$ and $\tau$ correspond to the normal and tangential components of the vectors to the surface $S$, the subscripts 1 and 2 correspond to adjacent me- 
dia with different electrophysical properties, and $\tau$ is any direction tangential to the surface of discontinuity [19]. For induced surface charge and current, the closing relations are absent [6] [7] [19]. The well-known Shchukin-Leontovich condition suggests that the properties of the medium vary slightly at distances of the order of the wavelength, but for rough surfaces this assumption leads to large errors in the calculations [6] [7] [18] [19].

\section{Generalized Wave Equation for the Intensity Vector and the Conditions at the Interfaces in the Presence of Strong Electromagnetic Field Breaks}

After some transformations of Formulas (4) and (5) we obtain

$$
\frac{\partial \boldsymbol{j}_{\mathrm{tot}}}{\partial t}=\frac{1}{\mu \mu_{0}} \nabla^{2} \boldsymbol{E}-\frac{1}{\mu \mu_{0}} \operatorname{grad}(\operatorname{div} \boldsymbol{E}) .
$$

In the Cartesian coordinates Formula (10) may be written as follows

$$
\begin{aligned}
& \frac{\partial j_{\text {tot } x}}{\partial t}=\frac{1}{\mu \mu_{0}}\left(\frac{\partial^{2} E_{x}}{\partial x^{2}}+\frac{\partial^{2} E_{x}}{\partial y^{2}}+\frac{\partial^{2} E_{x}}{\partial z^{2}}\right)-\frac{1}{\mu \mu_{0}} \frac{\partial}{\partial x}\left(\frac{\partial E_{x}}{\partial x}+\frac{\partial E_{y}}{\partial y}+\frac{\partial E_{z}}{\partial z}\right), \\
& \frac{\partial j_{\text {toty }}}{\partial t}=\frac{1}{\mu \mu_{0}}\left(\frac{\partial^{2} E_{y}}{\partial x^{2}}+\frac{\partial^{2} E_{y}}{\partial y^{2}}+\frac{\partial^{2} E_{y}}{\partial z^{2}}\right)-\frac{1}{\mu \mu_{0}} \frac{\partial}{\partial y}\left(\frac{\partial E_{x}}{\partial x}+\frac{\partial E_{y}}{\partial y}+\frac{\partial E_{z}}{\partial z}\right), \\
& \frac{\partial j_{\text {totz }}}{\partial t}=\frac{1}{\mu \mu_{0}}\left(\frac{\partial^{2} E_{z}}{\partial x^{2}}+\frac{\partial^{2} E_{z}}{\partial y^{2}}+\frac{\partial^{2} E_{z}}{\partial z^{2}}\right)-\frac{1}{\mu \mu_{0}} \frac{\partial}{\partial z}\left(\frac{\partial E_{x}}{\partial x}+\frac{\partial E_{y}}{\partial y}+\frac{\partial E_{z}}{\partial z}\right) .
\end{aligned}
$$

At the interface, the following relationship also holds [6]:

$$
\operatorname{div} \boldsymbol{i}_{\tau}+\lambda E_{x_{1}}-\lambda E_{x_{2}}=-\frac{\partial \sigma}{\partial t}
$$

We write conditions (6)-(9) in the Cartesian coordinate system

$$
\begin{aligned}
& D_{x_{1}}-D_{x_{2}}=\sigma, \\
& E_{y_{1}}-E_{y_{2}}=0, \\
& E_{z_{1}}-E_{z_{2}}=0, \\
& B_{x_{1}}-B_{x_{2}}=0, \\
& H_{y_{1}}-H_{y_{2}}=i_{z}, \\
& H_{z_{1}}-H_{z_{2}}=i_{y},
\end{aligned}
$$

where $\boldsymbol{i}_{\tau}=i_{y} \boldsymbol{j}+i_{z} \boldsymbol{k}$ is the surface current density.

The order of the system of differential Equations (11)-(13) is 18. By differentiating expression (15) whith respect to time and taking into account relation (14), we obtain the condition for the normal components of the total current at the interface:

$$
\operatorname{div} \boldsymbol{i}_{\tau}+j_{\mathrm{tot}_{1}}=j_{\text {totr }_{2}} .
$$

This allows us to exclude from consideration the surface charge density and surface current. The question is described in more detail in [6] [7] [19]. 
According to [6] [7] [19], the following conditions are met at the interface of adjacent media:

- the equality of the normal components of the total current;

- the equality of tangential projections of the electric field vortex;

- the conservation of electric charge;

- the equality of the tangential components of the electric field and their derivatives in the tangential direction;

- the equality of the derivatives of the normal components of the total current in the direction tangential to the interface of adjacent media, with account for the influence of surface currents without the introduction of a surface charge $\sigma$.

Thus, in each section of the layered medium, a numerical solution requires a use of through-counting schemes and, at the same time, sampling of the medium in such a way that the boundaries of layers could have common nodes. The medium was divided into finite elements so that the nodes of the finite-element grid lying on the interface simultaneously belonged to media with different electrophysical properties. In this case, the condition of equality of total currents, equality of charge fluxes [6] [7] [19] should be satisfied at the interface.

Reformulation of the Maxwell equations and their reduction to the wave equation allows one to exclude from consideration the surface charge and current [6] [7] [18] [19], but in this case, the number of boundary conditions increases and becomes equal to 15 . Such a task is technically difficult to solve, but if the conditions of the continuity of the total current and the through-counting scheme [18] [19] are used, the result can be obtained.

For example, in Comsol Multiphysics (or similar software), a splane simulated domain with different inclusions can be considered as one medium, where its properties depend on the coordinates $\varepsilon(r), \lambda(r)$. The boundary conditions are usually specified as $E_{x}=E_{x}(t) \sin \omega t, E_{y}=E_{t}(t) \cos \omega t$. This approach works well for both macroobjects and nanoobjects $10-50 \mathrm{~nm}$ in size, at a wavelength of about 400 - $500 \mathrm{~nm}$ (light) [6] [7].

When using Comsol Multiphysics, it is possible to obtain unreliable results, since the solution does not require compliance with the conditions at the corner points for the initial-boundary value problem. Therefore, for more accurate results, it is better to use Wolfram Mathematica.

It is known that the general solution of the wave equation is the d'Alembert solution-the sum of the forward and reverse waves, with the reverse wave having a broadening $\pm 2 \Delta$. As a result, for the electromagnetic wave we obtain

$$
\begin{aligned}
E_{x}(t) & =A[1+m \cos \theta t][2 \sin \omega t-\sin (\omega-2 \Delta) t+\sin (\omega+2 \Delta) t] \\
& =4 A[1 \pm m \cos \theta t] \sin ^{2} \Delta t \sin \omega t, \\
E_{y}(t) & =A[1+m \cos \theta t][2 \cos \omega t-\cos (\omega-2 \Delta) t+\cos (\omega+2 \Delta) t] \\
& =4 A[1 \pm m \cos \theta t] \sin ^{2} \Delta t \cos \omega t,
\end{aligned}
$$

The functions represented by Formulas (22) and (23) continuously fill the 
frequency range $\omega-2 \Delta \leq \omega \leq \omega+2 \Delta$. The broadening is caused by the interaction of the atom with the surrounding particles. In addition, the establishment of a signal at the boundary is a transitional process, so finally

$$
\begin{aligned}
E_{x}(t) & =A\left[\int_{\omega-2 \Delta}^{\omega+2 \Delta}\left(1-\frac{2}{\mathrm{e}^{\lambda_{0} \sin ^{2} \omega t}+\mathrm{e}^{-\lambda_{0} \sin ^{2} \omega t}}\right) \sin ^{2} \omega t \mathrm{~d} \omega\right] \frac{(1-m \cos \theta t) \sin \omega t}{4 \Delta} \\
& =\left[\int_{\omega-2 \Delta}^{\omega+2 \Delta}\left(1-\frac{2}{c h\left(\lambda_{0} \sin ^{2} \omega t\right)}\right) \sin ^{2} \omega t \mathrm{~d} \omega\right] \frac{(1-m \cos \theta t) \sin \omega t}{4 \Delta}, \\
E_{y}(t) & =A\left[\int_{\omega-2 \Delta}^{\omega+2 \Delta}\left(1-\frac{2}{\mathrm{e}^{\lambda_{0} \sin ^{2} \omega t}+\mathrm{e}^{-\lambda_{0} \sin ^{2} \omega t}}\right) \sin ^{2} \omega t \mathrm{~d} \omega\right] \frac{(1-m \cos \theta t) \sin \omega t}{4 \Delta} \\
& =\left[\int_{\omega-2 \Delta}^{\omega+2 \Delta}\left(1-\frac{2}{c h\left(\lambda_{0} \sin ^{2} \omega t\right)}\right) \sin ^{2} \omega t \mathrm{~d} \omega\right] \frac{(1-m \cos \theta t) \cos \omega t}{4 \Delta} .
\end{aligned}
$$

The quantities $E_{x}(t)$ and $E_{y}(t)$ satisfy the matching conditions $E_{x}(0)=0, E_{y}(0)=0, E_{x}^{\prime}(0)=0$, and $E_{y}^{\prime}(0)=0$, when radiation propagates with zero initial conditions.

\section{Mathematical Modeling}

\subsection{Building a Model}

We need to solve a mixed problem for a hyperbolic equation:

$$
\begin{gathered}
\lambda \frac{\partial E}{\partial t}+\varepsilon \varepsilon_{0} \frac{\partial^{2} E}{\partial t^{2}}=\frac{1}{\mu \mu_{0}}\left(\nabla^{2} E-\operatorname{grad}(\operatorname{div} E)\right), \\
\left.E\right|_{t=0}=0, \\
\left.\frac{\partial E}{\partial t}\right|_{t=0}=0, \\
E=\varphi(t) .
\end{gathered}
$$

Formula (26) is an equation for the electric field strength. We consider the case in 2D formulation, i.e. when the function $\boldsymbol{E}$ is two-dimensional. Formulas (27) and (28) represent zero initial conditions. Formula (29) is a boundary condition; $\Gamma$ is the boundary of the area where the solution is built. For simplicity of calculations and perception, we take a region of a square shape that has the limits $[0 ; 1] \times[0 ; 1]$.

The function $\varphi(t)$ carries information about the signal distributed in the area. Let us assume that the signal does not depend on the point of space in which it is located.

We rewrite the original problem for each of the components of the vector $E$, given that the boundary $\Gamma$ is square

$$
\begin{aligned}
& \lambda \frac{\partial E_{x}}{\partial t}+\varepsilon \varepsilon_{0} \frac{\partial^{2} E_{x}}{\partial t^{2}}=\frac{1}{\mu \mu_{0}}\left(\frac{\partial^{2} E_{x}}{\partial y^{2}}-\frac{\partial^{2} E_{y}}{\partial x \partial y}\right), \\
& \lambda \frac{\partial E_{y}}{\partial t}+\varepsilon \varepsilon_{0} \frac{\partial^{2} E_{y}}{\partial t^{2}}=\frac{1}{\mu \mu_{0}}\left(\frac{\partial^{2} E_{y}}{\partial y^{2}}-\frac{\partial^{2} E_{x}}{\partial x \partial y}\right),
\end{aligned}
$$




$$
\begin{gathered}
\left.E_{x}\right|_{t=0}=0,\left.\quad E_{y}\right|_{t=0}=0, \\
\left.\frac{\partial E_{x}}{\partial t}\right|_{t=0}=0,\left.\quad \frac{\partial E_{y}}{\partial t}\right|_{t=0}=0, \\
\left.E_{x}\right|_{x=0}=\varphi_{x}(t),\left.\quad E_{y}\right|_{x=0}=\varphi_{y}(t), \\
\left.E_{x}\right|_{x=1}=\varphi_{x}(t),\left.\quad E_{y}\right|_{x=1}=\varphi_{y}(t) \\
\left.E_{x}\right|_{y=0}=\varphi_{x}(t),\left.\quad E_{y}\right|_{y=0}=\varphi_{y}(t), \\
\left.E_{x}\right|_{y=1}=\varphi_{x}(t),\left.\quad E_{y}\right|_{y=1}=\varphi_{y}(t), \\
{[x ; y] \in[0 ; 1] \times[0 ; 1], \quad t \in\left[0 ; 5 \times 10^{-8}\right] .}
\end{gathered}
$$

The function $\boldsymbol{j}(t)=\left(\varphi_{x}(t), \varphi_{y}(t)\right)$ is described by the following equations [7]:

$$
\begin{aligned}
& \varphi_{x}(t)=\frac{1}{4 \Delta}\left[\int_{\omega-2 \Delta}^{\omega+2 \Delta}\left(1-\frac{2}{\mathrm{e}^{\lambda_{0} \sin ^{2} \varphi t}+\mathrm{e}^{-\lambda_{0} \sin ^{2} \varphi t}}\right) \sin ^{2} \varphi t \mathrm{~d} \varphi\right](1-m \cos \theta t) \sin \omega t, \\
& \varphi_{y}(t)=\frac{1}{4 \Delta}\left[\int_{\omega-2 \Delta}^{\omega+2 \Delta}\left(1-\frac{2}{\mathrm{e}^{\lambda_{0} \sin ^{2} \varphi t}+\mathrm{e}^{-\lambda_{0} \sin ^{2} \varphi t}}\right) \sin ^{2} \varphi t \mathrm{~d} \varphi\right](1-m \cos \theta t) \cos \omega t .
\end{aligned}
$$

The problem is modeled for $t \in\left[0 ; 5 \times 10^{-8}\right]$ due to the limited power of the computer. But this time is enough for the wave to cross the region several times. If computer resources were not limited, the problem can be solved for any $t \geq 0$.

The system of Equations (30)-(37) makes it possible to take into account the effect of induced surface electric charges at the interfaces of layered and angular structures [6] [7] [18] [19].

We recall that the problem is solved when the source region contains two media with different electromagnetic properties. For example, the propagated wave runs against a body that, of course, has a bounding surface. On a rather small scale, this surface represents roughness of different kind. Our task is to study and find out how roughness affects the electric field strength, and how strength is distributed in strong discontinuities at the boundary.

Based on integration with respect to the results obtained, we construct a continuous function by interpolation. It will be used as boundary conditions. The solution to this problem itself is obtained by applying the theory of finite-differences schemes and numerical methods. All this has been programmed in Wolfram Mathematica, and the necessary constants were taken from reference books.

\subsection{Graphic Visualization of the Results Obtained}

In this section we simulate electromagnetic field by using Formulas (30)-(37).

For clarity, we construct several rough surfaces that represent fractal.

The physical region is represented by a square of size $[0 ; 1] \times[0 ; 1]$. At the bottom of this region, we place the rough surfaces. As a result, the region ap- 
pears to by interface of two heterogeneous media, where strong discontinuities of the electromagnetic field are observed. The results of numerical simulation are presented in Figures 7-18. We also give examples of the propagation of electromagnetic waves modeled with the aid of the Comsol Multiphysics package. As already mentioned, the solution does not require the fulfillment of the agreement conditions at the corner points for the initial-boundary value problem, and the result can be considered only approximately. Moreover, the modeling of fractal surfaces is difficult to implement by means of this package. Therefore, we simulate a random roughness. Simulation results are presented below (see Figures 19-21).

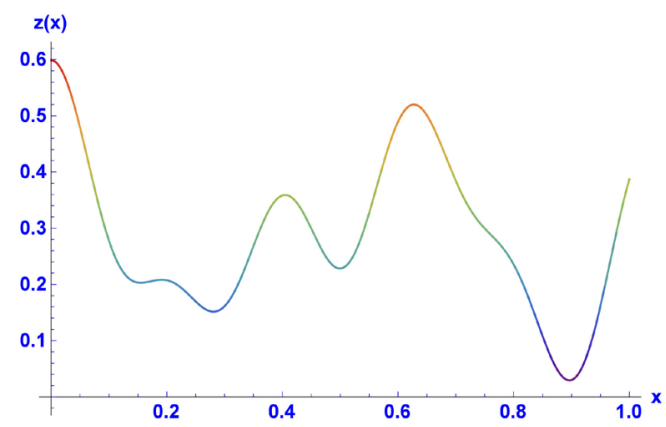

Figure 7. Fractal rough surface at $M=3, D_{f}=1.3, \gamma=1.7, L=2$ and $G=1$.

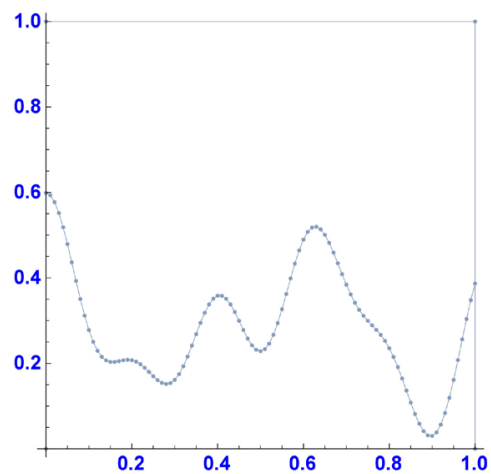

Figure 8. Discretization of the fractal rough surface for numerical calculations.
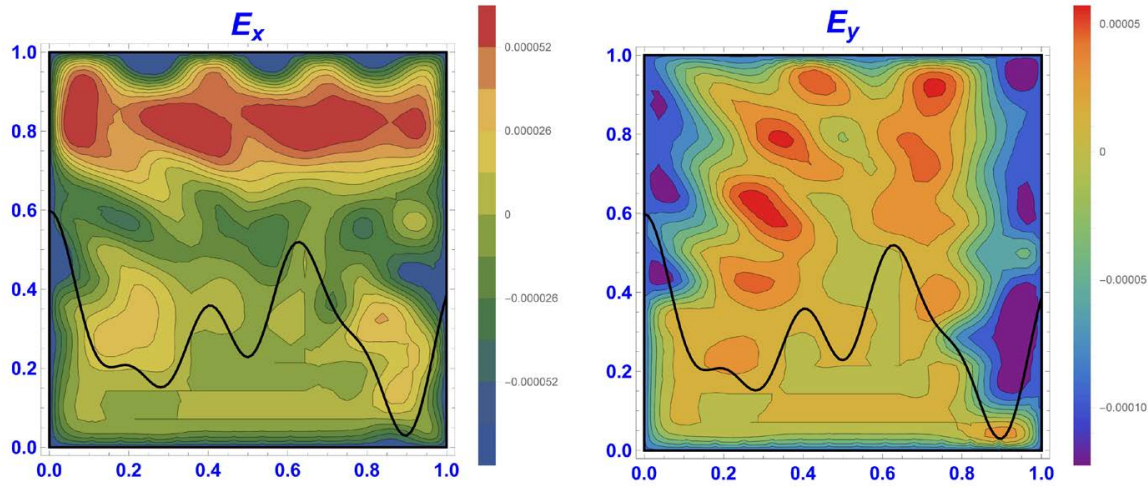

Figure 9. Vector of the electric field strength at $t=10^{-8}$. 


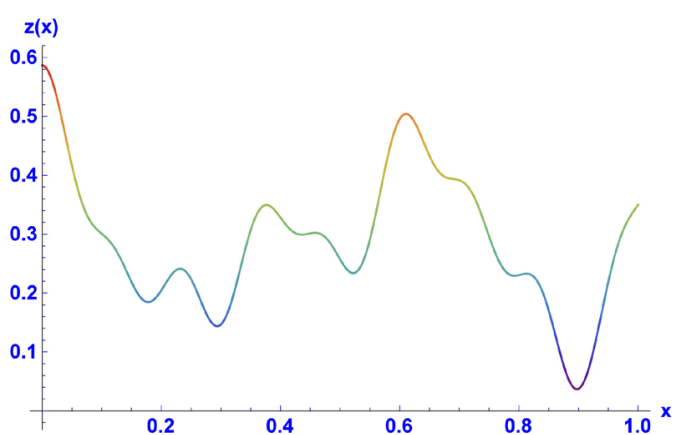

Figure 10. Fractal rough surface at $M=4, D_{f}=1.3, \gamma=1.7, L=3$ and $G=1$.

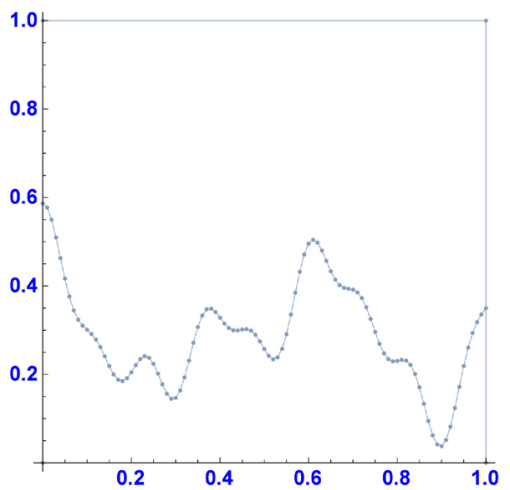

Figure 11. Discretization of the fractal rough surface for numerical calculations (the second surface).
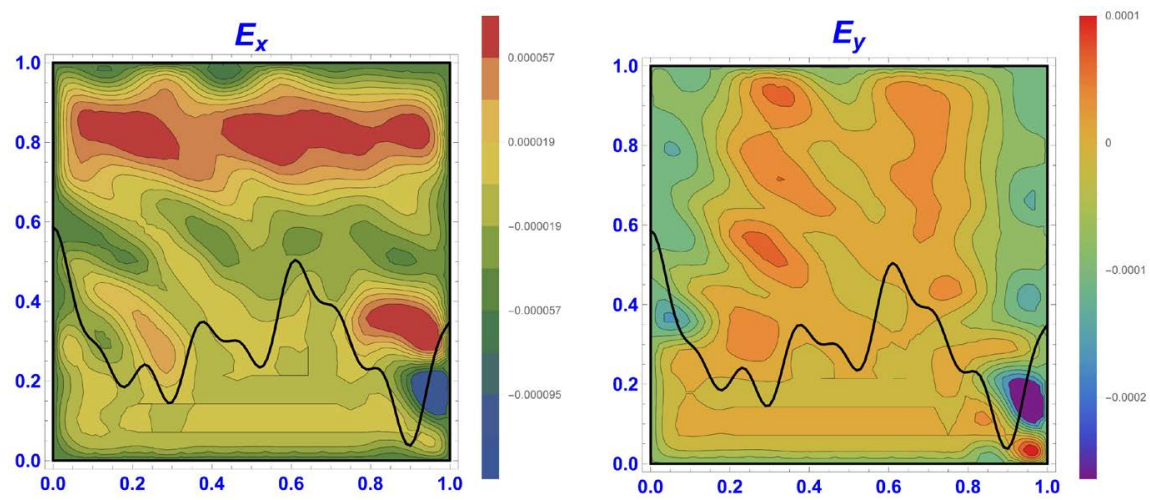

Figure 12. Vector of the electric field strength $t=10^{-8}$ for the second roughness.



Figure 13. Fractal rough surface at $M=2, D_{f}=1.3, \gamma=1.7, L=3$ and $G=1$. 


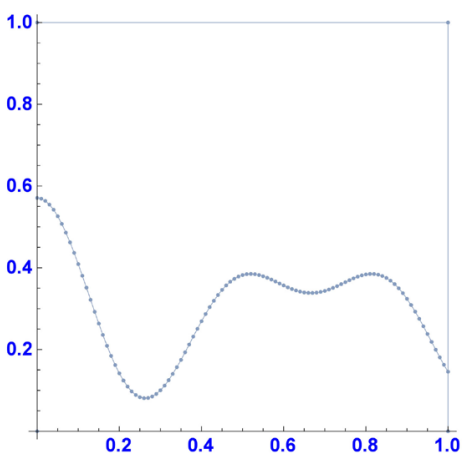

Figure 14. Discretization of the fractal rough surface for numerical calculations (the third surface).
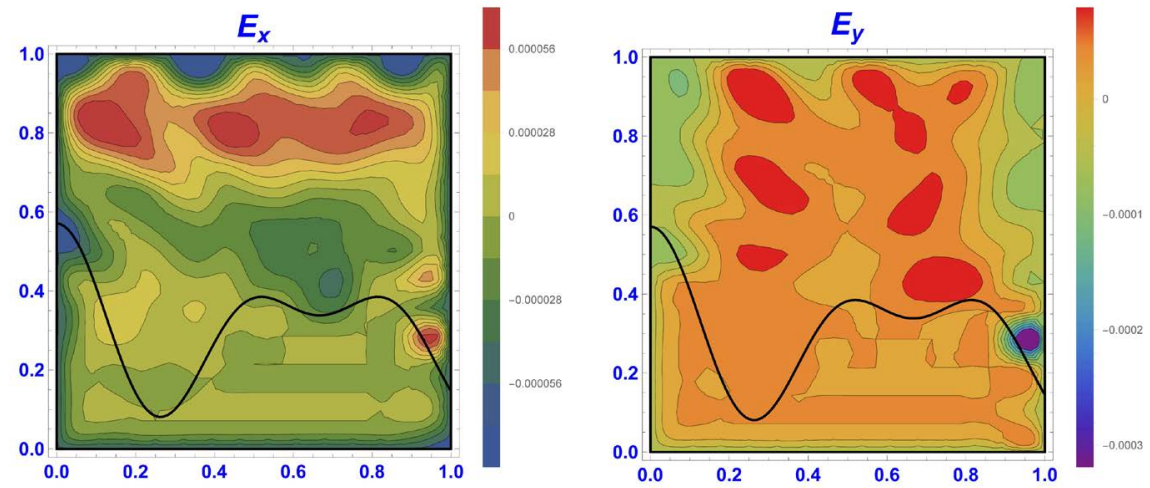

Figure 15. The vector of the electric field strength $t=10^{-8}$ for the third roughness.

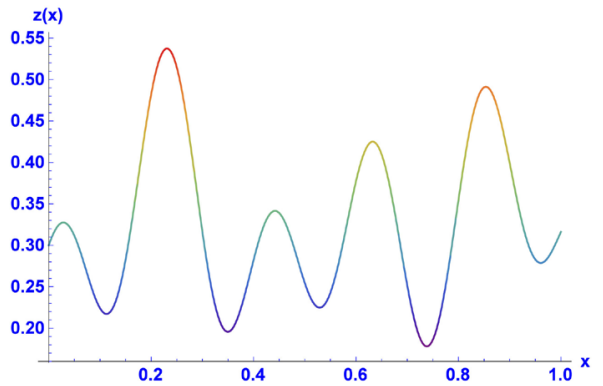

Figure 16. Fractal rough surface at $M=3, D_{f}=1.3, \gamma=1.7, L=3$ and $G=1$.

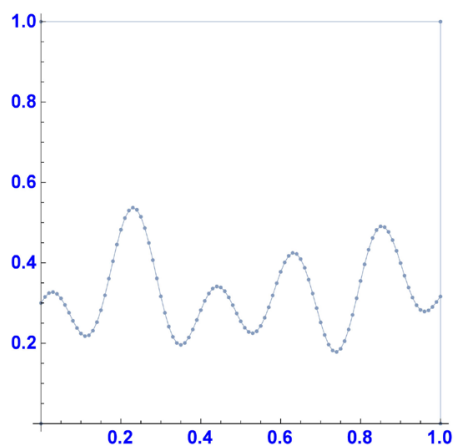

Figure 17. Discretization of the fractal rough surface for numerical calculations (the fourth surface). 

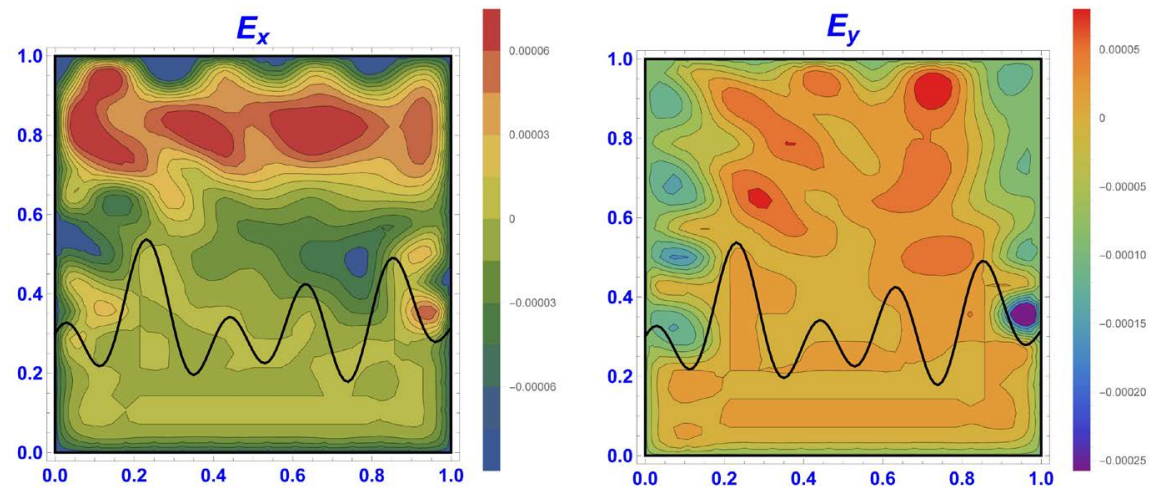

Figure 18. Vectors of the electric field strength $t=10^{-8}$ for the fourth roughness.

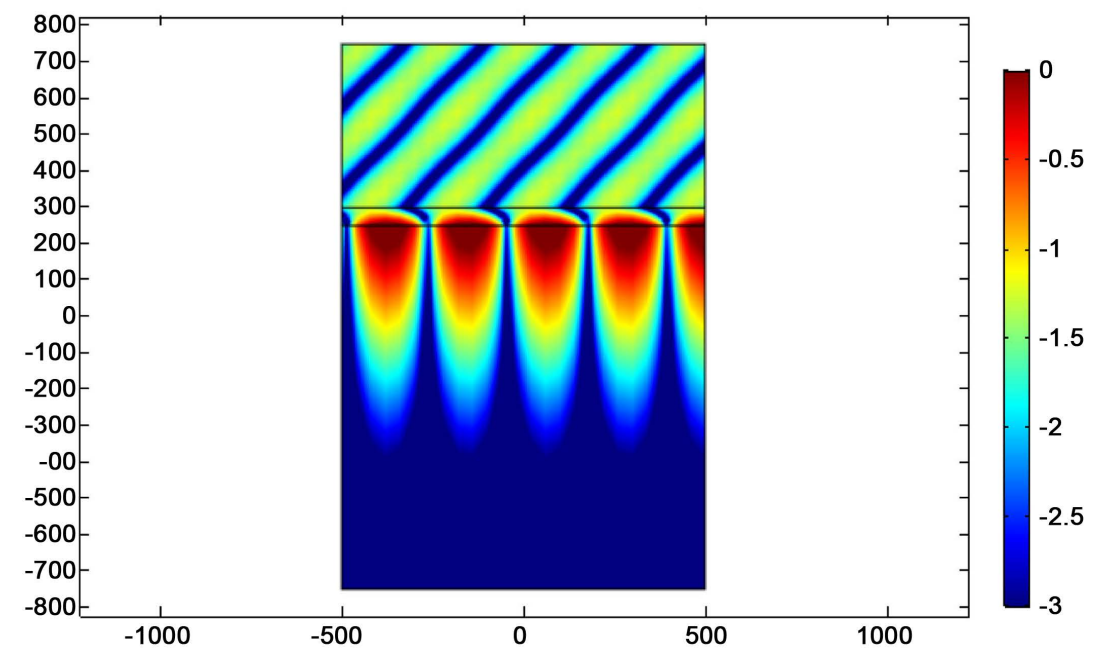

Figure 19. Distribution of the electromagnetic field energy. The size of the region is given in nanometers. The angle of incidence is $\bar{\theta}=40^{\circ}$, wavelength $\bar{\lambda}=475 \mathrm{~nm}$. The region includes a perfectly smooth silver plate and air.

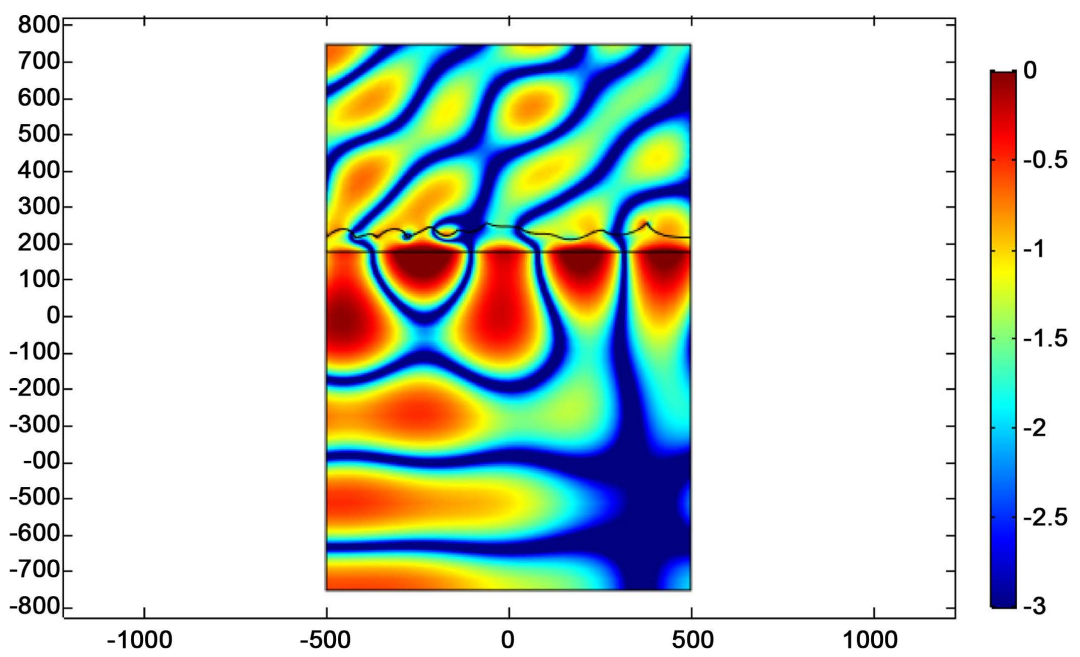

Figure 20. Distribution of the electromagnetic field energy. The size of the region is given in nanometers. The angle of incidence is $\bar{\theta}=40^{\circ}$, wavelength $\bar{\lambda}=475 \mathrm{~nm}$. The region includes a rough silver plate and air. 


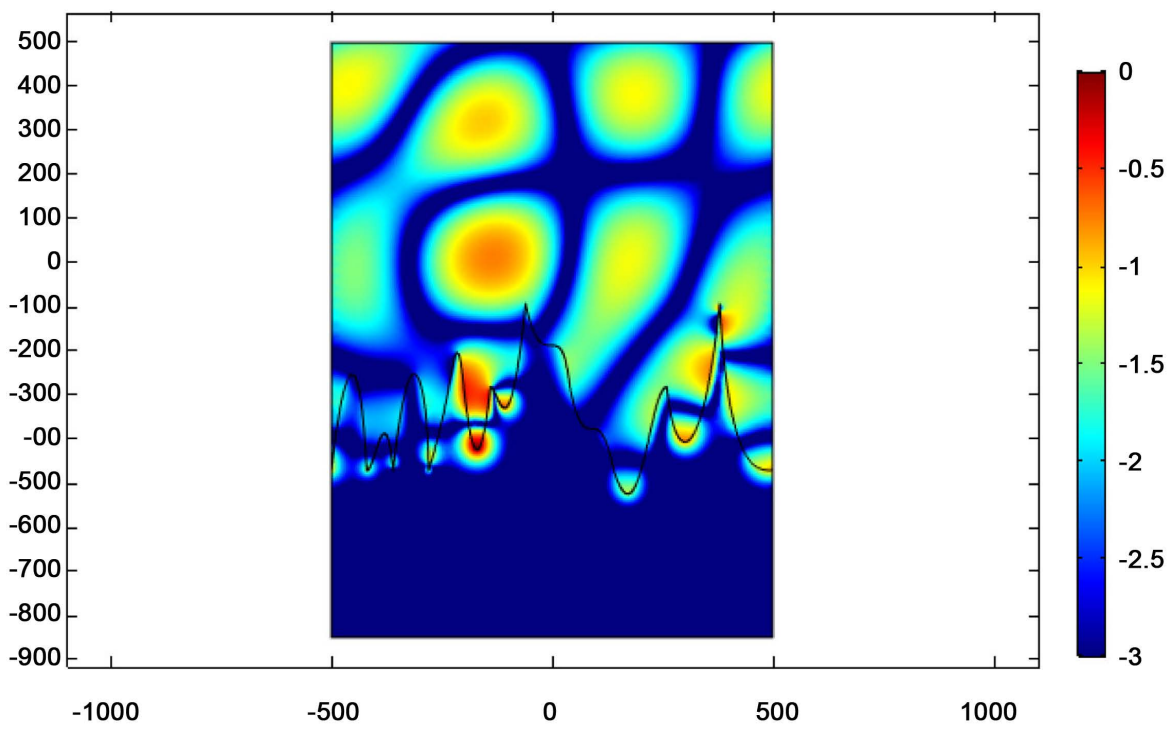

Figure 21. Distribution of the electromagnetic field energy. The size of the region is given in nanometers. The angle of incidence is $\bar{\theta}=40^{\circ}$, wavelength $\bar{\lambda}=475 \mathrm{~nm}$. The region represents the interface of the silver rough object and air.

\section{Conclusion}

A consistent physical-mathematical model of the propagation of electromagnetic waves in a heterogeneous system in the presence of strong electromagnetic field discontinuities at the interface between two media that represents a rough surface modeled using fractal geometry conception has been developed. Mathematical modeling of fractal rough surfaces using Weierstrass-Mandelbrot formulas was performed. The results obtained in the work in the form of constructed conditions for the continuity of the total current in the heterogeneous system make it possible to mathematically soundly approach to the use of end-to-end counting schemes in numerical simulations of the interaction of an electromagnetic field with piecewise homogeneous complex media. The presented results of numerical simulation show that the maximum intensity of the electromagnetic field is observed, due to nanofocusing, not on the edge of the roughness, but in its nearest vicinity. This approach can be applied to the problems of near-field optical microscopy and to the study of nanofocusing of light on the tip in nano-optics.

\section{Foundation}

The work was supported by the Belarusian Republican Foundation for Fundamental Research (Project F19-096 "electromagnetic wave propagation in inhomogeneous electromagnetic radiation shields").

\section{Conflicts of Interest}

The authors declare no conflicts of interest regarding the publication of this paper. 


\section{References}

[1] Barta, O., Pistora, I. and Vesec, I. (2001) Magnetooptics in Bi-Gyrotropic Garnet Waveguide. Opto-Electronics Review, 3, 320-325.

[2] Benett, J.M. (1985) Comparison of Techniques for Measuring the Roughness of Optical Surfaces. Optical Engineering, 24, 380-387.

https://doi.org/10.1117/12.7973493

[3] Dykhne, A.M. and Kasanova, I.M. (2002) The Leontovich Boundary Conditions and Calculation of Effective Impedance of Inhomogeneous Metal. Optics Communications, 206, 39-56. https://doi.org/10.1016/S0030-4018(02)01396-2

[4] Elson, J.M. and Bennett, J.M. (1979) Relation between the Angular Dependence of Scattering and the Statistical Properties of Optical Surfaces. Journal of the Optical Society of America, 69, 31-47. https://doi.org/10.1364/JOSA.69.000031

[5] Weber, V.L. (2017) Simulation of a Random Profile of the Sea Surface. Radiophysics and Quantum Electronics, 60, 309-322. https://doi.org/10.1007/s11141-017-9801-3

[6] Grinchik, N.N. (2014) Electrodynamics of Inhoogeneous (Laminated, Angular) Structures. Journal of Electromagnetic Analysis and Applications, 6, 57-105.

https://doi.org/10.4236/jemaa.2014.65009

[7] Grinchik, N.N., Andrejev, V.G., Zayats, G.M. and Grinchik, Y.N. (2018) Non-Monochromatic Electromagnetic Radiation of Inhomogeneous Media. Journal of Electromagnetic Analysis and Applications, 2, 13-33. https://doi.org/10.4236/jemaa.2018.102002 http://www.scirp.org/journal/jemaa

[8] Larruquert, J.I. (2001) Reflectance Enhancement with Sub-Quarterwave Multilayers of Highly Absorbing Materials. Journal of the Optical Society of America B, 6, 1406-1414. https://doi.org/10.1364/JOSAA.18.001406

[9] Monzon, I.I., Yonte, T. and Sanchez-Soto, L.L. (2003) Characterizing the Reflectance of Periodic Lasered Media. Optics Communications, 218, 43-47. https://doi.org/10.1016/S0030-4018(03)01192-1

[10] Red'ko, V.P., Khomchenko, A.V. and Erevich, V.A. (2003) Automodulation of Laser Radiation Reflected from a Two-Cavity Resonance Medium. Doklady Natsionalnoi Akademii Nauk Belarusi, 1, 57-61. (In Russian)

[11] Sedrakin, D.H., Gevorgyan, A.H. and Khachatrian, A.Zh. (2001) Transmission of Plane Electromagnetic Wave Obliquely Incident on a One-Dimensional Isotropic Dielectric Medium with an Arbitrary Reflective Index. Optics Communications, 195, 1-9. https://doi.org/10.1016/S0030-4018(01)01153-1

[12] Simonsen, I., Vanderbrouoq, D. and Roux, S. (2001) Electromagnetic Wave Scattering from Conducting Self-Affine Surfaces: An Analytic and Numerical Study. Journal of the Optical Society of America B, 5, 1101-1111. https://doi.org/10.1364/JOSAA.18.001101

[13] Agranovich, V.M. (1985) Surface Polaritons. Science, Moscow. (In Russian)

[14] Volkov, Y. M. (1962) Plasma in a Magnetic Field and Direct Conversion of Thermal Energy into Electrical Energy. Gosatomizdat, Moscow. (In Russian)

[15] Veiko, V.P. and Dyshlovenko, S.S. (2001) Laser Microstructuring of Surfaces. Journal of the St. Petersburg National Research University of Information Technologies, Mechanics and Optics, 4, 119-128. (In Russian)

[16] Libenson, M.N., Yakovlev, E.B. and Shandybina, G.D. (2014) Interaction of Laser Radiation with a Substance: Textbook: At 3 Parts. Part 1: Mechanisms of Absorption and Dissipation of Energy in a Substance. St. Petersburg State University 
ITMO, St. Petersburg. (In Russian)

[17] Goldstein, L.D. and Grains, N.V. (1971) Electromagnetic Waves. 2nd Edition, Nauka, Moscow.(In Russian)

[18] Grinchik, N.N., Zhuk, V.A., Khmyl, A.A. and Tsurko, V.A. (2000) Interaction of Thermal and Electrical Phenomena in Polarized Media. Mathematical Modelling, 11, 67-76. (In Russian)

[19] Grinchik, N.N. (2008) Modeling of Electrophysical and Thermophysical Processes in Layered Media. Nat. Acad. Science of Belarus, A.V. Luikov Institute of Heat and Mass Transfer. Belaruskaya Nauka, Minsk. (In Russian)

[20] Biryukov, V.V., Grachev, V.A. and Lobin, S.G. (2018) Round Shielded Waveguide with a Rough Innersurface. Antennas, 10, 54-63. (In Russian)

[21] Degtyarev, S.A., Ustinov, A.V. and Honina, S.N. (2014) Nanofocusing with Pointed Structures. Computer Optics Journal, 4, 629-637. (In Russian) https://doi.org/10.18287/0134-2452-2014-38-4-629-637

[22] Demkin, N.B. (1970) Contacting a Rough Surface. Nauka, Moscow. (In Russian)

[23] Leontovich, M.A. (1948) On the Approximate Boundary Conditions for the Electromagnetic Field on the Surface of Well-Conducting Bodies. Radio Wave Propagation Studies. Ed. B.A. Vvedensky, Moscow, Part 2. (In Russian)

[24] Libenson, M.N. (1996) Surface Electromagnetic Waves in the Optical Range. Soros Educational Journal, 10, 92-98.

[25] Maryshev, S.N. and Shevyahov, N.S. (2010) Electromagnetic Surface Waves at the Boundaries of a Relativistic Moving Plasma Layer. Journal of Radio Electronics, 2, 1-21. (In Russian) http://jre.cplire.ru/jre/feb10/2/text.html

[26] Nazvanov, V.F. (2015) Surface Electromagnetic Waves of the Optical Range (Plasmon-Polaritons): Properties, Application. Journal "News of Saratov University. New Series". Physics Series, 1, 5-14. (In Russian)

[27] Ogar, P.M. and Gorokhov, D.B. (2010) Contact Interaction of Rough Surfaces: A Fractal Approach. Systems Methods Technology. Bratsk State University, 6, 30-38. (In Russian)

[28] Perfilyev, I. (2014) Surface Roughness. Kinds, Methods and Parameters. Blog about Metal Quality Control [Electronic Resource]. (In Russian) http://ndt-welding.com/sherohovatost-poverhnosti-vidyi-metodyi-i-parametryi/

[29] Petrin, A.B. (2016) Patterns of Nanofocusing of a Surface Plasmon Wave in the Vicinity of the Nano-Apex of a Metallic Microtip. Teplofizika vysokih temperatur, 4 , 500-507. (In Russian)

[30] Samarskii, A.A. (1989) Theory of Difference Schemes. Nauka, Moscow. (In Russian)

[31] Sviridenok, A.I., Chizhik, S.A. and Petrokovets, M.I. (1990) Mechanics of Discrete Friction Contact. Nauka and Tekhnika, Minsk. (In Russian)

[32] Toporets, A.S. (1988) Rough Surface Optics. Mashinostroenie, Leningrad. (In Russian)

[33] Shvets, V.A. and Spesivtsev, E.V. (2013) Ellipsometry: A Teaching Aid for Laboratory Work. NSU Publishing House, Novosibirsk. (In Russian)

[34] Schneider, Yu.G. (2001) Operational Properties of Parts with a Regular Microrelief. St. Petersburg HITMO, St. Petersburg. (In Russian) 